\title{
Saúde mental em tempos de pandemia COVID-19: uma perspetiva da Medicina
}

\author{
https://doi.org/10.21814/uminho.ed.24.1
}

\section{Pedro Morgado}

Pedro Morgado (ORCID: 0000-0003-3880-3258) é Vice-Presidente da Escola de Medicina da Universidade do Minho e professor de Psiquiatria e Comunicação Clínica da mesma Escola. É investigador do domínio de Neurociências, no ICVS e médico Psiquiatra no Hospital de Braga. 


\section{O IMPACTO INICIAL}

A COVID-19, doença provocada pelo novo coronavírus SARS-CoV-2, foi declarada pandemia pela Organização Mundial de Saúde (OMS) no dia 11 de março de 2020. Desde que o surto inicial foi identificado no final de $2019 \mathrm{em}$ Wuhan, na China, tinham sido detetados mais de 118 mil casos de infeção em 114 países e 4.291 mortes.

Em Portugal, os primeiros dois casos foram confirmados a 2 de março. No dia 8 de março, um surto com epicentro na Freguesia de Idães, concelho de Felgueiras, encerrou o campus de Gualtar da Universidade do Minho, a Escola EB 2,3 de Idães e o edifício partilhado pelo Instituto de Ciências Biomédicas Abel Salazar e pela Faculdade de Farmácia da Universidade do Porto.

Nos dias seguintes, as notícias que chegavam de Itália, primeiro, e de Espanha, depois, geravam preocupação entre todos. Enquanto isso, o Conselho Nacional de Saúde Pública defendeu a manutenção das escolas em funcionamento presencial e a adoção de medidas de contenção muito limitadas quando comparadas com o que sucedia noutros países europeus. A 12 de março de 2020, o Conselho de Escolas Médicas Portuguesas dirige uma carta aberta ao Primeiro-Ministro em que questiona a "capacidade técnica" do Conselho Nacional de Saúde Pública e apela a medidas mais restritivas para evitar o colapso da resposta do sistema de saúde (Wong, 2020). Umas horas depois, o governo anuncia o encerramento das escolas a partir de 16 de março.

No dia 18 de março, o Presidente da República declarou o Estado de Emergência em todo o território. Com esta declaração, foram implementadas restrições à circulação de pessoas, tendo sido recomendado o confinamento no domicílio exceto para atividades consideradas essenciais, como trabalho autorizado, deslocaçóes à farmácia e aos supermercados e, ainda, a prática de exercício físico por períodos limitados.

De um dia para o outro quase tudo mudou. Pediram-nos que permanecêssemos confinados durante praticamente todo o tempo. Uma grande parte das pessoas passou a trabalhar a partir de casa, preferencialmente por telefone e computador. As consultas médicas não urgentes foram desmarcadas ou substituídas por uma chamada telefónica. Pediram-nos que mudássemos completamente grande parte dos nossos hábitos. 
E nós também mudámos. Enquanto vivenciamos as diferentes fases da pandemia (que ainda não foi ultrapassada) experimentamos diferentes respostas psicológicas: o medo de contrair o vírus, o receio de ter familiares afetados, a tristeza provocada pelo isolamento e pela mudança de planos importantes, a incerteza em relação ao fim do confinamento, a ansiedade provocada pela possível falha nas medidas de proteção individual, a saudade provocada pela distância física dos familiares, o luto pela perda de familiares e/ou amigos, a apreensão em relação aos impactos sociais e económicos da crise e o medo do aumento da violência familiar levaram a que muitas pessoas tivessem experienciado sintomas de stress, ansiedade ou medo (Salema, 2020).

Esses sintomas surgem pela ativação do sistema biológico de resposta ao stress - um sistema significativamente apurado ao longo da evolução das espécies que tem como finalidade garantir a ativação rápida de uma resposta fisiológica normal aos eventos anormais que podem colocar em risco o equilíbrio do organismo. O sistema de resposta ao stress tem como objetivo imediato a mobilização rápida dos recursos e da energia que forem necessários para a proteção da integridade do organismo e, como objetivo diferido, a criação de memórias que permitam respostas proporcionadas em situaçôes futuras (Morgado et al., 2017).

Quando uma ameaça é percecionada (por exemplo, o ataque por parte de um predador) desencadeia-se uma resposta rápida que prepara o indivíduo para a luta ou fuga (em inglês flight or fight) através da libertação de grandes quantidades de catecolaminas (como a adrenalina) na medula da glândula adrenal. A par da sobreativação psicológica, verifica-se um aumento da frequência cardíaca e da frequência respiratória, acompanhado por uma redistribuição do fluxo sanguíneo que privilegia o suprimento dos músculos dos membros inferiores (fundamentais para a fuga aos predadores) em detrimento do sistema digestivo. Esta resposta aguda ao stress é geralmente positiva, preparando o organismo para a ação e melhorando a performance (Morgado et al., 2017).

Em paralelo, o organismo inicia uma resposta mais lenta que é mediada pela libertação de glucocorticoides pelo córtex da glândula adrenal. Esta resposta tem como objetivo reestabelecer as condições fisiológicas do organismo, atenuando as respostas imunológica, inflamatória e endocrinológica ao stress. É mediada pelo eixo 
hipotálamo-pituitária-adrenais (HPA) e participa no processo de geração de memórias associadas ao evento (Morgado et al., 2017).

Estes mecanismos de resposta ao stress evoluíram ao longo de milhões de anos para preservar os indivíduos das ameaças à sua integridade física, sendo igualmente ativados quando se verifica uma exposição aos stressores modernos de natureza psicológica. Isso explica que várias pessoas tenham experienciado palpitações ou desconforto gástrico quando leram notícias ou informações acerca da disseminação da pandemia e dos seus impactos brutais na nossa vida comum.

Num mundo intensamente digital, uma medida indireta dos níveis de stress pode ser obtida através da análise do comportamento das pessoas na internet. Um estudo sobre as publicações em português e em inglês relacionadas com a COVID-19 de utilizadores de Portugal na rede social Twitter detetou um pico de expressóes relacionadas com medo entre os dias 6 e 8 de março, precisamente no momento em que eclodiam os primeiros focos significativos no país e se suspendiam as atividades letivas presenciais no campus de Gualtar da Universidade do Minho e em duas faculdades da Universidade do Porto (Trifan et al., 2020).

As respostas mediadas pelo sistema do stress dominaram os primeiros momentos do impacto da pandemia. O comportamento individual (e, por consequência, o devir coletivo) foi aqui determinado por uma rápida (e naturalmente sujeita a erros) avaliação dos riscos que levou à adoção de alguns comportamentos extremados por excesso e por defeito.

Os profissionais de saúde e todos os trabalhadores das funções essenciais viram-se repartidos entre o ímpeto de preservação pessoal e a imperiosidade do serviço público. Mas, ao contrário do que sucedera em catástrofes de outros tempos, o cunho humanista que caracteriza a nossa sociedade ficou patente na atempada proteção dos mais vulneráveis: pessoas com idade avançada (superior a 65 anos), doença cardíaca, doença pulmonar, doença oncológica, hipertensão arterial, diabetes e com sistema imunitário comprometido, foram especialmente protegidas desde a primeira hora, salvaguardando-se a sua condição de risco sempre que possível. 


\section{O CONFINAMENTO}

O segundo momento da resposta à pandemia inicia-se com a declaração do Estado de Emergência pelo Presidente da República. Nesta fase, os direitos dos cidadãos são significativamente restringidos, apelando-se para o cumprimento do estrito confinamento no domicílio. As saídas apenas são autorizadas para atividades consideradas essenciais, como deslocações para o trabalho, farmácia ou supermercado. Ao contrário do que sucedeu noutros países, como em Espanha, as saídas para a prática de exercício físico individual durante curtos períodos são autorizadas.

Nesta fase, as deslocações reduzem-se drasticamente como comprova a análise dos dados de geolocalização das operadoras nacionais de rede móvel (Melo et al., 2020) e da Google COVID-19 Community Mobility Reports (Tamagusko \& Ferreira, 2020). A análise destes dados de mobilidade ilustra a rapidez da adoção das regras de confinamento por parte dos portugueses.

Atendendo aos riscos de contaminação associados a alguns procedimentos médicos e ao receio da sobrecarga - e mesmo rutura - do Serviço Nacional de Saúde (SNS), a maioria dos atos médicos do SNS são suspensos e substituídos por consultas telefónicas. Os hospitais e consultórios do setor privado adaptaram a sua resposta, tendo encerrado inúmeros serviços e instalações.

A resposta da comunidade foi um sucesso. Em quatro semanas, Portugal era notícia nos meios de comunicação social internacional pela forma rápida como tinha implementado as medidas que lhe permitiram achatar a curva de crescimento do número de infeçôes (Ames, 2020; Jones, 2020) e divergir do sucedido em Espanha e Itália.

Do ponto de vista da literacia em saúde, um estudo realizado demonstrou diferenças no nível de conhecimento acerca da doença, dos seus modos de transmissão e das medidas de proteção entre os utentes de um Centro de Saúde do Nordeste Transmontano (Silva et al., 2020). As pessoas mais jovens, do género feminino e com maiores níveis de instrução apresentavam melhores conhecimentos acerca da doença.

Mas o confinamento não foi fácil. De acordo com um estudo realizado na Europa e Estados Unidos da América verificou-se um aumento significativo das buscas 
realizadas no motor de pesquisa Google por palavras relacionadas com tédio e solidão após o início do confinamento obrigatório (Brodeur et al., 2020).

Ao longo das diferentes semanas do confinamento, a nossa equipa de investigação efetuou um estudo longitudinal que avaliou semanalmente a saúde mental de um grupo de mais de mil voluntários portugueses e 400 voluntários espanhóis. No início do Estado de Emergência, verificaram-se níveis elevados de stress, ansiedade, sintomas de depressão e sintomas obsessivo-compulsivos (Moreira et al., 2020). Estes dados estão em linha com os verificados noutros países europeus atingidos pela pandemia e que experienciaram situações de confinamento obrigatório.

Este estudo permitiu identificar alguns fatores protetores e de vulnerabilidade para o desenvolvimento de sintomas relacionados com o stress, ansiedade, depressão e obsessivo-compulsivos. Assim, as pessoas do género feminino, as pessoas desempregadas, as que ficaram sem trabalho devido à pandemia e aquelas que consomem mais de uma hora por dia de informação acerca da COVID-19 apresentaram valores mais elevados de ansiedade, stress e depressão. Em sentido contrário, a prática de exercício físico regular, a existência de um jardim na habitação e a idade avançada revelaram-se fatores protetores em termos de saúde mental (Moreira et al., 2020). Convém realçar que o grupo de pessoas com idade avançada que participaram neste estudo não foi representativo da população de Portugal com mais de 65 anos, apresentando-se mais escolarizado do que a média nacional. Ao contrário do verificado noutros estudos, o número de elementos do agregado familiar e a existência de um animal doméstico não se revelaram fatores preditores em termos de saúde mental.

No mesmo estudo participaram 204 pessoas com doença psiquiátrica prévia. Deste grupo, aqueles que mantiveram as suas consultas com recurso a tecnologia digital apresentaram significativamente melhores indicadores em termos de stress, ansiedade, depressão e sintomas obsessivo-compulsivos (Moreira et al., 2020).

Ao longo das semanas, os níveis de stress e ansiedade desceram de forma consistente com duas exceçôes: entre as pessoas desempregadas, os níveis mantiveram-se altos; entre os homens, os níveis mantiveram-se baixos. Mulheres e pessoas que trabalham (presencialmente ou à distância) reduziram significativamente os seus níveis de 
sofrimento (Bento, 2020a). Em termos de qualidade do sono e satisfação com a qualidade de vida, verificou-se uma diminuição sustentada dos indicadores em todos os grupos estudados (Bento, 2020a).

Os profissionais de saúde, em particular os médicos, apresentaram valores mais elevados de stress, ansiedade e depressão do que a população geral. Quando se comparou entre médicos da linha da frente e médicos que não consideravam estar na linha da frente verificámos que os médicos da linha frente apresentavam níveis mais elevados de stress, ansiedade, depressão e sintomas obsessivo-compulsivos (Bento, 2020b). Para fazer face ao expectável aumento de situações de doença psiquiátrica e sofrimento mental entre os profissionais, a Escola de Medicina da Universidade do Minho em conjunto com o Programa Nacional para a Saúde Mental da DGS, a Ordem dos Médicos, a Sociedade Portuguesa de Psiquiatria e Saúde Mental e a Associação Portuguesa de Internos de Psiquiatria desenvolveu o programa "Cuidar de Quem Cuida”, um serviço gratuito de consultas de Psiquiatria para profissionais de saúde, garantido por mais de 250 psiquiatras voluntários e assegurado através do Centro de Medicina Digital P5.

As restrições impostas à participação nas cerimónias fúnebres durante a vigência do Estado de Emergência configuram uma situação particularmente relevante pelo potencial impacto negativo na saúde mental. Estas restriçôes impossibilitaram o cumprimento dos rituais tradicionais e limitaram o número de participantes nos funerais, reduzindo a rede de suporte e dificultando o processo de luto (Aguiar et al., 2020).

No seu conjunto, estes dados reforçam dois factos muito relevantes. Em primeiro lugar, e ao contrário do que tem sido sistematicamente repetido, esta crise pandémica não afeta a todos por igual: as pessoas que sofrem de doença psiquiátrica, as pessoas que não têm trabalho e aquelas que têm menor suporte social e mais baixos recursos financeiros estão em maior risco de sofrimento psíquico e de desenvolvimento de uma doença psiquiátrica. Curiosamente, os fatores económico-sociais, como a pobreza, também se constituem como fatores de risco per se para a mortalidade por COVID-19 (Williamson et al., 2020), factos que nos alertam para a consequência das desigualdades mesmo em tempo de pandemia. Em segundo, as consultas médicas por chamada telefónica devem ser utilizadas de forma complementar e não sistemática, de modo a garantir que os cuidados são prestados de acordo com o 
necessário. Além do mais, a perceção de que uma chamada telefónica pode substituir uma consulta está longe de ser algo consensual entre os utentes portugueses.

\section{O DESCONFINAMENTO}

O terceiro momento da resposta à pandemia COVID-19 em Portugal iniciou-se com o levantamento do Estado de Emergência. Nesta fase, o medo de um vírus desconhecido foi substituído pela imperiosidade de reduzir a transmissão para evitar o surgimento de novos picos e pelo receio de uma crise social e económica à escala global.

Nesta fase, as respostas do SNS não diretamente relacionadas com a COVID-19 recomeçaram a ser disponibilizadas aos utentes. O ritmo de retoma não permitiu repor todos os atos médicos cancelados durante o Estado de Emergência, fase em que se verificou uma situação de excesso de mortalidade 3,5 a 5 vezes superior ao explicado pelas mortes COVID-19 reportadas oficialmente (Nogueira et al., 2020). Os hospitais e outros serviços de saúde privados retomaram a sua atividade assistencial no cumprimento das normas e informações da DGS.

A esmagadora maioria das pessoas irá adaptar-se de forma saudável, recuperando integralmente os níveis de funcionamento prévios. Mesmo após situações de catástrofe significativamente mais devastadoras, a maioria das pessoas não desenvolve qualquer psicopatologia. No entanto, algumas pessoas podem experienciar o surgimento e/ou agravamento de doenças psiquiátricas.

Quando ativados de forma contínua ou excessiva, os mecanismos de resposta ao stress podem tornar-se prejudiciais. O stress crónico pode favorecer o desenvolvimento de doenças psiquiátricas como a depressão, as perturbaçóes de ansiedade, a doença obsessivo-compulsiva ou a perturbação de stress pós-traumático.

Como mencionado, os riscos não se distribuem de forma simétrica na população, sendo possível identificar pessoas com maior vulnerabilidade para o desenvolvimento de doenças psiquiátricas. Assim, os grupos mais vulneráveis em termos de saúde mental incluem: (1) as pessoas que contraem a COVID-19, em particular, aquelas que apresentam fatores de risco (idade superior a 65 anos, doença cardíaca, respiratória ou oncológica e imunossupressão); (2) as pessoas com doença psiquiátrica prévia; (3) as 
pessoas que sofrem de dependências; (4) as pessoas vítimas e/ou em risco de violência doméstica; (5) os profissionais de saúde; (6) os imigrantes e as pessoas que integram grupos étnicos, culturais e sexuais minoritários; (7) as pessoas em situação económico-social desfavorável, nomeadamente as pessoas sem-abrigo; (8) os trabalhadores do sexo; e (9) as pessoas com idade avançada, nomeadamente quando vivam isoladas (Kawohl \& Nordt, 2020, p. 19; Office of the United Nations High Commissioner for Human Rights, 2020; Pfefferbaum \& North, 2020; Platt et al., 2020).

A prevenção do suicídio deve constituir uma prioridade em termos de saúde mental (Gunnell et al., 2020; Kawohl \& Nordt, 2020). O grupo colaborativo para a investigação em prevenção de suicídio COVID-19 propôs um conjunto de estratégias de redução do risco de suicídio em tempo de pandemia COVID-19 que inclui: (1) o adequado e atempado funcionamento dos serviços de psiquiatria e saúde mental; (2) a existência de estruturas de suporte para a crise suicidária aguda (como linhas de apoio); (3) a redução dos impactos financeiros da pandemia através da implementação de políticas públicas de alimentação, habitação e emprego; (4) a garantia de apoio e proteção às vítimas de violência doméstica; (5) o desenvolvimento de políticas públicas de redução do consumo de álcool; (6) a promoção de grupos comunitários que visem reduzir o isolamento social e a solidão; (7) a garantia do acesso aos bens essenciais durante a pandemia; (8) e a promoção de comportamentos responsáveis (não alarmistas nem sensacionalistas) por parte dos órgãos de comunicação social (Gunnell et al., 2020).

Em Portugal, a situação de jogo deve ser monitorizada com particular atenção tendo em conta os elevados níveis de gastos em raspadinhas que têm sido descritos ao longo dos últimos anos (Vilaverde \& Morgado, 2020).

A fase de desconfinamento representa uma adaptação das pessoas à nova normalidade que nos irá acompanhar durante um período incerto de tempo. Nesta fase, a implementação de estratégias de promoção da saúde mental é fundamental para reduzir o impacto da curva de doenças psiquiátricas que tem sido sistematicamente anunciada (Tabela 1). 
Tabela 1. Níveis de intervenção em saúde mental em tempo de pandemia COVID-19.

Prevenção (comunitária)

A maioria das pessoas poderá

experienciar sintomas de stress
Redução do consumo de notícias

Procura de fontes fiáveis de informação

Exercício físico regular

Padrão regular de sono e apetite

Manter interações sociais (mesmo à distância)

Psicoeducação

Evitar substâncias aditivas e jogos de sorte e azar

Psicoeducação

Ferramentas de autoavaliação da saúde mental

Ferramentas online de gestão emocional

Intervenções precoces breves

Linhas de apoio psicológico

O diagnóstico de doença psiquiátrica foi estabelecido e requer intervenção
Consultas de Medicina Geral e Familiar

Consultas de Psiquiatria

Consultas de Psicologia

Este tipo de estratégias de promoção da saúde mental deve implementar-se em níveis simultaneamente decrescentes em termos de abrangência e crescentes em termos de personalização e diferenciação. Assim, as medidas de prevenção especificamente implementadas para responder aos impactos da pandemia na saúde mental, deverão ser complementadas por um amplo programa de diagnóstico e de intervenção breve. Às pessoas com diagnóstico estabelecido de doença psiquiátrica deve ser providenciado o acesso a cuidados médicos em contexto de Medicina Geral e Familiar e/ou no âmbito dos Serviços Locais de Saúde Mental.

Considerando os impactos descritos nas fases anteriores, a pandemia COVID-19 representa uma oportunidade para a implementação e melhoria dos serviços digitais na prestação de cuidados de saúde, em particular no âmbito da Psiquiatria. Em sentido lato, a Medicina Digital já faz parte das práticas clínicas da maioria dos profissionais de saúde. A realização de chamadas em tempo real e a troca de SMS e e-mails é uma prática 
recorrente embora frequentemente desvalorizada pelos modelos de gestão em vigor na maioria dos serviços do SNS (Torous et al., 2020).

Ao longo da pandemia tem resultado evidente que o potencial da prestação de cuidados de saúde com mediação digital requer um nível de preparação e adequação que ainda não foi globalmente atingido pelos serviços do SNS. Em concreto, é necessário garantir formação aos profissionais de saúde para a realização de consultas em contexto digital e, em simultâneo, melhorar a infraestrutura informática dos serviços do SNS para garantir a possibilidade de realizar consultas a distância por videochamada, a modalidade de interação digital que melhor protege a relação terapêutica, transmitindo ao utente uma tranquilidade assinalável.

Tabela 2. Tipos de interação em Medicina Digital.

Interações

Síncronas

Interaçôes Assíncronas

Monitorização Remota
Consultas por telefone em tempo real

Videochamadas em tempo real

Utilização de estetoscópios digitais, otoscópios ou ecógrafos através de um assistente à distância

Envio de mensagens, imagens ou informação para resposta posterior (e-mail; chat; SMS; outros)

Ferramentas online de gestão emocional

Educação para a Saúde e/ou Literacia em Saúde

Transmissão de medições fisiológicas do paciente para o prestador de cuidados (frequência cardíaca, tensão arterial, glicemia, acelerómetro, etc.)

Uma massificação da utilização de meios digitais nas consultas ditada por razôes epidemiológicas, ainda que temporária, de psiquiatria e de saúde mental poderá ser dificultada pela impossibilidade de realização de um exame físico adequado, pela maior dificuldade em abordar tópicos sensíveis e pela possível rejeição da tecnologia por parte de alguns utentes e profissionais. No entanto, os seus benefícios suplantam largamente as dificuldades potenciais e incluem: (1) a possibilidade de providenciar cuidados urgentes em situações que não necessitem de presença física no consultório; (2) a promoção de cuidados não urgentes e/ou de seguimento a utentes conhecidos, evitando 
deslocaçốes; (3) a garantia de suporte e treino aos utentes com doenças crónicas (gestão do peso, informação dietética, etc.); (4) a prestação de cuidados de saúde física e ocupacional; (5) a monitorização de sinais de doenças crónicas; (6) a acessibilidade aos cuidados para utentes que se encontram em regióes distantes e remotas; (7) a garantia do acompanhamento após hospitalização; (8) o planeamento de cuidados e o aconselhamento em situações de crise/urgência; (9) a promoção de cuidados de saúde não emergentes em lares de idosos (particularmente relevante durante COVID-19); (10) a formação e o treino a prestadores de cuidados de saúde que se encontrem distantes.

\section{CONCLUSÃO}

A pandemia COVID-19 atingiu a nossa sociedade e modificou o nosso modo de vida de uma forma que ninguém poderia imaginar quando começou o ano de 2020. A resposta psicológica e comportamental é, globalmente, adaptativa e tendente à implementação de estratégias que visem a manutenção da homeostasia e, consequentemente, a integridade e a sobrevivência.

Quando surgiram os primeiros sinais de alarme, as respostas psicológicas foram dominadas pela preocupação e pelo stress, tendo-se verificado níveis variáveis de desorganização comportamental e prejuízo na tomada de decisão. Na fase subsequente de confinamento, as respostas emocionais mais típicas poderiam englobar o medo, a solidão e a ansiedade. Nas situaçóes em que falharam os mecanismos de controlo, o stress crónico pode evoluir para queixas persistentes de depressão, ansiedade, perturbação obsessivo-compulsiva e perturbação de sono.

No essencial, a crise acentuou o melhor e o pior das sociedades, afetando de forma brutalmente desigual as pessoas que compóem o nosso tecido social. Aqueles que se encontraram numa situação de maior vulnerabilidade no início da pandemia foram os que sofreram as consequências mais severas desta nova normalidade. Em sentido contrário, aqueles que usufruem de melhores condiçôes sociais e materiais desenvolveram estratégias de coping globalmente mais adaptativas, experienciando sintomas de sofrimento mental mais ligeiros. 
No momento em que a sociedade se mobilizou de forma ímpar, o SNS deve promover as respostas entendidas como adequadas para enfrentar as próximas fases da pandemia. A incorporação da tecnologia contribuirá para uma medicina mais digital e mais apta para responder aos desafios de uma nova quarentena. Ao SNS compete gerar as condições necessárias para que as pessoas com doença psiquiátrica não se vejam privadas do necessário acompanhamento em consultas de psiquiatria e de psicologia.

A missão da Escola de Medicina da Universidade do Minho é melhorar os cuidados de saúde através da formação, da investigação e da geração de valor. Ao investigar e caracterizar a saúde mental durante o confinamento, ao promover o voluntariado médico, ao dinamizar serviços de prestação de cuidados de saúde, ao fomentar a literacia e ao desenhar ferramentas de autoavaliação e de gestão emocional, a Escola de Medicina da Universidade do Minho cumpriu a sua missão em tempos inusitadamente estranhos e difíceis. Mais do que contributos avulsos, estes projetos agregam os componentes que uma resposta em saúde mental não pode deixar de incluir.

\section{REFERÊNCIAS BIBLIOGRÁFICAS}

Aguiar, A., Pinto, M., \& Duarte, R. (2020). Grief and Mourning during the COVID-19 Pandemic in Portugal. Acta Médica Portuguesa, 33(13), Article 13. https://doi.org/10.20344/amp.14345.

Ames, P. (2020, April 14). How Portugal became Europe's coronavirus exception. POLITICO. https://www. politico.eu/article/how-portugal-became-europes-coronavirus-exception/.

Bento, H. (2020a, May 28). Há boas notícias sobre o estado da nossa ansiedade e más sobre a qualidade do sono: Um estudo do confinamento. Jornal Expresso. https://expresso.pt/coronavirus/2020-05-28-Ha-boasnoticias-sobre-o-estado-da-nossa-ansiedade-e-mas-sobre-a-qualidade-do-sono-um-estudo-do-confinamento.

Bento, H. (2020b, June 19). Saúde Mental. Bastonário pede “plano de recuperação” para médicos na linha da frente contra a COVID - E teme abandonos da profissão. Jornal Expresso. https://expresso.pt/sociedade/202006-19-Saude-Mental.-Bastonario-pede-plano-de-recuperacao-para-medicos-na-linha-da-frente-contra-aCOVID---e-teme-abandonos-da-profissao.

Brodeur, A., Clark, A., Fleche, S., \& Powdthavee, N. (2020). COVID-19, Lockdowns and Well-Being: Evidence from Google Trends (SSRN Scholarly Paper ID 3596670). Social Science Research Network. https:/papers. ssrn.com/abstract $=3596670$. 
Gunnell, D., Appleby, L., Arensman, E., Hawton, K., John, A., Kapur, N., Khan, M., O’Connor, R. C., Pirkis, J., Appleby, L., Arensman, E., Caine, E. D., Chan, L. F., Chang, S.-S., Chen, Y.-Y., Christensen, H., Dandona, R., Eddleston, M., Erlangsen, A., ... Yip, P. S. (2020). Suicide risk and prevention during the COVID-19 pandemic. The Lancet Psychiatry, 7(6), 468-471. https://doi.org/10.1016/S2215-0366(20)30171-1.

Jones, S. (2020, April 19). Swift action kept Portugal's coronavirus crisis in check, says minister. The Guardian . http://www.theguardian.com/world/2020/apr/19/swift-action-kept-portugals-coronavirus-crisis-in-checksays-minister.

Kawohl, W., \& Nordt, C. (2020). COVID-19, unemployment, and suicide. The Lancet Psychiatry, 7(5), 389390. https://doi.org/10.1016/S2215-0366(20)30141-3.

Melo, H.P. M., Henriques, J., Carvalho, R., Verma, T., da Cruz,J. P., \& Araujo, N. A. M. (2020). Heterogeneous impact of a lockdown on inter-municipality mobility. ArXiv:2006.15724 [Physics, q-Bio]. http://arxiv.org/ abs/2006.15724.

Moreira, P. S., Ferreira, S., Couto, B., Machado-Sousa, M., Fernandez, M., Raposo-Lima, C., Sousa, N., PicoPerez, M., \& Morgado, P. (2020). Protective elements of mental health status during the COVID-19 outbreak in the Portuguese population. MedRxiv, 2020.04.28.20080671. https://doi.org/10.1101/2020.04.28.20080671.

Morgado, P., Cerqueira, J. J., \& Sousa, N. (2017). Resposta neuronal ao stresse. In Neurociências (pp. 401412). Lidel.

Nogueira, P. J., Nobre, M. de A., Nicola, P. J., Furtado, C., \& Carneiro, A. V. (2020). Excess Mortality Estimation During the COVID-19 Pandemic: Preliminary Data from Portugal. Acta Médica Portuguesa, 33(6), 376-383. https://doi.org/10.20344/amp.13928.

Office of the United Nations High Commissioner for Human Rights. (2020). COVID-19 and the Human Rights of LGBTI People. https://www.ohchr.org/Documents/Issues/LGBT/LGBTIpeople.pdf.

Pfefferbaum, B., \& North, C. S. (2020). Mental Health and the COVID-19 Pandemic. New England Journal of Medicine, Aug 6;383(6):510-512, null. https://doi.org/10.1056/NEJMp2008017.

Platt, L., Elmes, J., Stevenson, L., Holt, V., Rolles, S., \& Stuart, R. (2020). Sex workers must not be forgotten in the COVID-19 response. Lancet (London, England), 396(10243), 9-11. https://doi.org/10.1016/S01406736(20)31033-3.

Salema, I. (2020, April 5). Saúde mental: "A incerteza quanto ao fim do isolamento é um claríssimo factor de risco.” PÚBLICO. https://www.publico.pt/2020/04/05/ciencia/noticia/saude-mental-incertezafim-isolamento-clarissimo-factor-risco-1910502.

Silva, J. G. da, Silva, C. S., Alexandre, B., \& Morgado, P. (2020). Health literacy of inland population in the mitigation phase 3.2. Of COVID-19's pandemic in Portugal - A descriptive cohort study. MedRxiv, 2020.05.11.20098061. https://doi.org/10.1101/2020.05.11.20098061.

Tamagusko, T., \& Ferreira, A. (2020). Mobility patterns of the Portuguese population during the COVID-19 pandemic. https://arxiv.org/abs/2007.06506v1. 
Torous, J., Myrick, K. J., Rauseo-Ricupero, N., \& Firth, J. (2020). Digital Mental Health and COVID-19: Using Technology Today to Accelerate the Curve on Access and Quality Tomorrow. JMIR Mental Health, 7(3), e18848. https://doi.org/10.2196/18848.

Trifan, A., Matos, S., Morgado, P., \& Oliveira, J. L. (2020). Bilingual emotion analysis in social media throughout the COVID-19 pandemic in Portugal. https://openreview.net/forum?id=KXptqXxP6oO.

Vilaverde, D., \& Morgado, P. (2020). Scratching the surface of a neglected threat: Huge growth of Instant Lottery in Portugal. The Lancet Psychiatry, 7(3), e13. https://doi.org/10.1016/S2215-0366(20)30039-0.

Williamson, E. J., Walker, A. J., Bhaskaran, K., Bacon, S., Bates, C., Morton, C. E., Curtis, H. J., Mehrkar, A., Evans, D., Inglesby, P., Cockburn, J., McDonald, H. I., MacKenna, B., Tomlinson, L., Douglas, I. J., Rentsch, C. T., Mathur, R., Wong, A. Y. S., Grieve, R., Goldacre, B. (2020). OpenSAFELY: Factors associated with COVID-19 death in 17 million patients. Nature, 1-11. https://doi.org/10.1038/s41586-020-2521-4.

Wong, B. (2020, March 12). Escolas Médicas apelam a Costa para que tome "medidas restritivas o mais precocemente possível.” PÚBLICO. https://www.publico.pt/2020/03/12/sociedade/noticia/escolas-medicasapelam-costa-tome-medidas-restritivas-precocemente-possivel-1907511. 